\title{
BMJ Open Interventions for pregnant women who use tobacco and other substances: a systematic review protocol
}

\author{
Melissa A Jackson (D) , ${ }^{1,2}$ Amanda L Baker (D) , ${ }^{1}$ Kristen L McCarter (D) , ${ }^{1}$ \\ Amanda L Brown (D) ,1,2 Gillian S Gould (D) , ${ }^{1}$ Adrian J Dunlop (D) ${ }^{1,2,3}$
}

To cite: Jackson MA, Baker AL, McCarter KL, et al. Interventions for pregnant women who use tobacco and other substances: a systematic review protocol. BMJ Open 2019;9:e032449. doi:10.1136/ bmjopen-2019-032449

- Prepublication history and additional material for this paper are available online. To view these files, please visit the journal online (http://dx.doi. org/10.1136/bmjopen-2019032449).

Received 19 June 2019 Revised 02 September 2019 Accepted 26 September 2019

Check for updates

(C) Author(s) (or their employer(s)) 2019. Re-use permitted under CC BY-NC. No commercial re-use. See rights and permissions. Published by BMJ.

${ }^{1}$ School of Medicine and Public Health, University of Newcastle, Newcastle, New South Wales, Australia

${ }^{2}$ Drug and Alcohol Clinical Services, Hunter New England Local Health District, Newcastle, New South Wales, Australia

${ }^{3}$ Drug \& Alcohol Clinical

Research \& Improvement Network, Sydney, New South Wales, Australia

Correspondence to

Melissa A Jackson;

Mel.Jackson@health.nsw.gov.au

\section{ABSTRACT}

Introduction The prevalence of tobacco smoking in pregnancy remains elevated in some disadvantaged populations of women. One group is those who use alcohol and/or other psychoactive substances during pregnancy, with tobacco use prevalence estimates ranging from $71 \%$ to $95 \%$. Although effective evidence-based cessation treatments exist, few women with co-occurring substance use problems successfully stop smoking during pregnancy. There is limited information about treatments that specifically target this group and a summary of the available research is required to assist and enhance the development of innovative cessation interventions. This article describes a protocol for a comprehensive review of studies that have trialled behavioural and/or pharmacological tobacco cessation interventions in populations of pregnant women who are nicotine dependent and use alcohol and/or other psychoactive substances.

Methods and analysis The review will undertake literature searches in MEDLINE, PsycINF0, CINAHL, EMBASE and ProQuest databases, as well as the grey literature. Studies of any design methodology will be included if they describe changes to tobacco smoking behaviours in quantitative terms. No restriction on year of publication or published language will apply. Participants include pregnant women of any age, who smoke tobacco, who are seeking or having treatment, or in post-treatment recovery for the use of psychoactive substances. Interventions are any psychological, behavioural or pharmacological treatments used to treat tobacco use. Outcome measures are any that quantitatively report abstinence or reductions in participant tobacco consumption. Key details and tobaccorelated outcomes from included studies will be extracted and tabulated before being narratively synthesised. The systematic review protocol has been developed according to the Preferred Reporting Items for Systematic Reviews and Meta-Analyses Protocols guidelines.

Ethics and dissemination Ethics approval is not required. Findings will be disseminated via peer-reviewed literature, conference presentations, media and social media.

PROSPERO registration number CRD42018108777

\section{INTRODUCTION}

Tobacco smoking during pregnancy is the major modifiable contributor to adverse maternal, fetal and neonatal outcomes. ${ }^{12}$ Maternal smoking during pregnancy has been strongly associated with intrauterine growth

\section{Strengths and limitations of this study}

- This protocol was designed according to the Preferred Reporting Items for Systematic Reviews and Meta-Analyses for Protocols guidelines.

- The review will be strengthened methodologically by independent double screening and data extraction and the use of the Effective Public Health Practice Project quality assessment tool.

- A broad search strategy has been devised, including a search of the grey literature, to capture all possible trialled interventions that fall within the scope of this review.

- The review may be limited by the heterogeneity of study methodologies and outcome measures that restrict the ability to pool outcome data and assess the effectiveness of cessation treatments.

restriction, ectopic pregnancy, placental abruption, placenta praevia, preterm birth, miscarriage and stillbirth. ${ }^{34}$ Infants exposed to prenatal tobacco smoking are more likely to experience low birth weight, sudden unexpected death in infancy, chronic respiratory disorders, cardiovascular disease, obesity, attachment difficulties, learning and behavioural difficulties and have a greater likelihood of developing tobacco and other substance use disorders later in life. ${ }^{4-9}$

The prevalence of maternal tobacco smoking has declined significantly worldwide since $1985 .{ }^{10}$ Estimates from 2016 suggest a global prevalence of smoking during pregnancy of $1.7 \%$ (95\% CI $0.0 \%$ to $4.5 \%)$, ranging from $0.8 \%(95 \%$ CI $0.0 \%$ to $2.2 \%)$ in the African region to $8.1 \%$ (95\% CI $4.0 \%$ to $12.2 \%$ ) in the European region. ${ }^{10}$ Despite the declines in general maternal populations, elevated rates of tobacco use in pregnancy remain in some overlooked, but high-risk groups of women. One group in particular, is women who use alcohol and other psychoactive substances (including opioids, cannabis, stimulants and benzodiazepines) during pregnancy. Although population-wide 
smoking rates for this group are hard to find, published prevalence estimates from studies of pregnant women in substance use treatment range from $71 \%$ to $95 \% .^{11-14}$ Women who describe using multiple substances are also more likely to smoke tobacco than those who use only one (OR: $2.35,95 \%$ CI 1.37 to 4.04$){ }^{14}$

Pregnancy is typically viewed as a period of high motivation to cease tobacco use, driven by a protective urge to safeguard the fetus. ${ }^{15}$ A large number of effective, evidence-based treatments exist for the general population of pregnant women to assist cessation, reflecting the significant public health concerns that surround the issue. A 2017 Cochrane review ${ }^{16}$ assessed 102 psychosocial interventions for women to stop smoking in pregnancy that addressed the mental, social or emotional factors related to nicotine dependence. Moderate-quality evidence found that these interventions increased the proportion of women who stopped smoking in late pregnancy by $35 \%$ when compared with controls. ${ }^{16}$ Such interventions include incentive-based programme, motivational interviewing, cognitive-behavioural therapy, health education, social support and biochemical feedback on maternal and fetal nicotine exposure. ${ }^{13}$

Pharmacotherapies are also available to support maternal tobacco cessation. Nicotine replacement therapy (NRT), varenicline and bupropion and more recently, electronic nicotine delivery systems (ENDS; eg, electronic cigarettes) used for the purpose of stopping smoking are commonly used in general populations. The combination of NRT with behavioural strategies has been shown to improve cessation outcomes when compared with outcomes from single interventions or usual care, ${ }^{17}$ and is considered gold standard for tobacco treatment in general populations. ${ }^{18}$ The evidence is not as clear for pregnant women, with a 2015 Cochrane review of pharmacotherapies for smoking cessation in pregnancy finding marginal support for NRT delivered with behavioural support, suggesting that it may increase smoking abstinence by $40 \%$ in late pregnancy (Risk Ratio 1.43 , 95\% CI 1.03 to 1.93$){ }^{19}$ This borderline evidence may be due to the dosages of NRT trialled being unable to counter the increased metabolism of nicotine that occurs during pregnancy ${ }^{20}$ and recommendations for higher doses to be used in future antenatal smoking cessation studies have been made. ${ }^{19}$ Support for the use of ENDS, buproprion or varenicline as an aid for cessation is limited in pregnancy, with the same 2015 review reporting only one small trial of bupropion and none of varenicline or ENDS. ${ }^{19}$

Yet, despite the availability of treatments and their effectiveness in general maternal populations, there are few pregnant women with co-occurring tobacco and substance use disorders who successfully abstain from tobacco. Poor cessation outcomes do not appear to be associated with a lack of desire to quit. Pregnant women with substance use disorders have the same urges to protect their unborn children and aspire to stop tobacco consumption at rates similar to those who do not use substances. ${ }^{142122}$ Regrettably, a range of barriers impact their ability to quit and contribute to poor outcomes when attempting to stop using currently available cessation treatments. ${ }^{142123-31}$

Physiologically, pregnant women with substance use disorders are more likely to have a comorbid mental illness, consume greater amounts of tobacco and experience more severe nicotine withdrawal symptoms than those without substance use disorders. ${ }^{30}$ Smoking tobacco is also known to enhance the psychoactive effects of opioids and cannabis ${ }^{23} 25$ while counteracting some of the adverse cognitive effects of alcohol consumption. ${ }^{26}{ }^{28}$ Socially, the cultural norms associated with tobacco smoking in this population, ${ }^{24}$ and the high proportion of women with partners and/or other household members who smoke, ${ }^{1421}$ have significant impacts on cessation efforts. Systemic barriers occur at a treatment level because priority is often given to cessation of alcohol or other drug use over tobacco use during pregnancy. ${ }^{27} 31$ The high level of support required of healthcare professionals to facilitate tobacco cessation in this group ${ }^{14}$ is often prohibited by organisational and individual factors, including lack of knowledge, time, confidence and counselling skills. ${ }^{29}$

The absence of effective tobacco smoking treatments for pregnant women with other substance use problems and the need for aggressive targeted efforts to reduce smoking in this high-risk group has been documented. ${ }^{13} 3233$ A 2014 systematic review of tobacco treatments for pregnant women receiving opiate agonist treatment found three published studies. ${ }^{13}$ Only one, a randomised controlled trial conducted in 2012 ( $n=102)$, had a significant, positive effect on smoking abstinence. ${ }^{34}$ A 2011 comparative study $(n=91)$, significantly influenced reduction in tobacco use but not abstinence ${ }^{35}$ and a 2004 randomised controlled trial $(\mathrm{n}=63)$ achieved increases in motivation to stop smoking but had no impact on cessation rates. ${ }^{36}$ The review had no constraints on time since publication, but was limited to interventions for pregnant women receiving opiate agonist treatment, omitting those targeting women with other substance use disorders.

A 2011 review of interventions designed to reduce or eliminate tobacco smoking during pregnancy uncovered two studies from a possible 97 that specifically targeted substance use populations. ${ }^{33}$ The results included one of the aforementioned studies. ${ }^{36}$ The other, a 1996 pilot study $(n=34)$, implemented a compulsory smoking treatment in a smoke-free in-patient maternal substance use programme. ${ }^{37}$ The intervention increased motivation to stop smoking but did not assess reductions in tobacco use or cessation. This review focused on smoking treatment interventions for all pregnant women, grouping by population and only reporting studies that met predetermined ratings for methodological rigour and quality. The review was restricted to a 20-year time frame (1990-2010) and focused on research from Canada and the USA.

The current evidence suggests that there is a shortage of smoking cessation treatments targeting pregnant women with substance use disorders. Given that smoking prevalence is now greatest in groups vulnerable to social 
disadvantage, including those with substance use disorders, ${ }^{38}$ and that treatment targets are now focusing on disparities in smoking rates between population groups, ${ }^{39}$ more innovative and comprehensive cessation interventions are acutely needed. To support the future development of such treatments, an appraisal of approaches that have previously assisted smoking cessation in maternal substance use populations, including those that have not significantly impacted abstinence, is required.

Therefore, the current review will focus on providing the most comprehensive synthesis of smoking cessation treatments for pregnant women with concurrent substance and tobacco use to date. This will be achieved by including all interventions that have been trialled in this group, regardless of participant demographic, substance of concern or time since studied.

\section{Objectives}

This review will systematically examine all studies that have trialled behavioural and/or pharmacological tobacco cessation interventions in populations of pregnant women who are nicotine dependent and use alcohol and/or other psychoactive substances. It will provide an updated summary of the evidence to date and highlight those interventions that have demonstrated effectiveness in the reduction of, or abstinence from, tobacco use in this high-priority group of women.

\section{METHODS}

The review protocol follows the recommendations outlined in the Preferred Reporting Items for Systematic Reviews and Meta-Analyses for Protocols (PRISMA-P) statement, ${ }^{40}$ and the review findings will be reported using published PRISMA guidelines. ${ }^{41}$

\section{Criteria for study inclusion}

Study characteristics

Studies must offer a treatment designed to reduce or stop tobacco smoking in pregnant women with substance use concerns. For the purpose of this review, tobacco encompasses all combustible products including cigarettes, cigars, pipes and hookahs. Treatments that target a combination of tobacco and cannabis use will be included but those that specifically target cannabis smoking will not.

Studies of any design methodology will be included, provided that they report quantitative outcomes relating to changes in tobacco smoking behaviours. Designs may be experimental or quasi-experimental, including prepost interventions, pilot and feasibility studies. There will be no limit on year of report publication and studies may be published in any language. Abstracts written in a language other than English will be translated using Google Translate to assess eligibility. The full text of those meeting the criteria will be professionally translated. Preclinical or animal studies will be excluded.

\section{Participants}

Participants may be pregnant women of any age, who smoke tobacco. They must be seeking or having treatment, or in post-treatment recovery for the use of alcohol and/ or other psychoactive substances.

\section{Interventions}

Interventions to be included may be any psychological, behavioural or pharmacological treatments used to treat tobacco dependence, including the use of ENDS to reduce or aid smoking cessation. Examples may include, but are not limited to, counselling therapies such as motivational interviewing and cognitive behavioural therapy, contingency management, social support or self-help strategies and pharmacotherapies including NRT and electronic cigarettes. They may be offered in health- or communitybased settings and mode of delivery may be in-person or remotely using telecommunication technologies.

\section{Outcomes}

Outcome measures will include any measure that reports abstinence or reductions in tobacco consumption of participants. These may be biochemical validation measures or self-reported measures, with or without biochemical verification. Examples of biochemical validation measures include carbon monoxide and cotinine (usually urinary or saliva), while self-report measures may include, but are not limited to, prolonged abstinence, point prevalence abstinence, continuous abstinence or number of cigarettes smoked. ${ }^{42}$

\section{Information sources}

\section{Electronic databases}

Literature searches for relevant published articles will be performed in the following databases: MEDLINE, PsycINFO, CINAHL and EMBASE. The ProQuest database will be searched to identify appropriate dissertations and theses. The search strategy will include MeSH terms and keywords associated with tobacco use, pregnancy, psychoactive substance use and interventions for smoking cessation. An example of a search created for MEDLINE is available as online supplementary material. Identical search terms will be adopted for each of the remaining databases, with changes to syntax made as required.

\section{Other sources}

The following strategies will be used to obtain studies not identified through electronic database searches:

i. A manual search of the reference lists of included full-text articles.

ii. A grey literature search using Google Scholar and reviewing the first 20 pages of results.

iii. Contacting experts in the specific field about research currently being conducted or unpublished study results.

\section{Data management and collection}

All identified titles and abstracts will be stored in Covidence, an electronic screening and data extraction tool recommended for Cochrane reviews. ${ }^{43}$ Two reviewers (MJ and KM) will independently screen articles against eligibility criteria to determine inclusion status, with a 
comparison of results made to ensure consistency. Full articles of included abstracts will then be reviewed against the inclusion criteria by the same reviewers. Any discrepancies at either stage will be discussed and referred to a third reviewer for consensus where necessary. Articles deemed ineligible will be documented, with the reason for exclusion noted for acknowledgement in the full review.

\section{Data extraction}

All full text articles remaining after the screening process will have relevant data extracted independently by two reviewers (MJ and $\mathrm{KM})$. A data extraction template will be developed based on recommendations by the Cochrane Handbook for Systematic Reviews of Interventions. ${ }^{44}$ The template will be piloted by both reviewers on the initial two articles and amendments made if necessary.

The following data variables to be extracted include:

- Author(s), journal and year.

- Study type, recruitment setting, study design, country, recruitment setting and strategy.

- Eligibility criteria, participant demographic and clinical characteristics including primary substance consumed.

- Intervention characteristics including strategies used, delivery method, duration and follow-up.

- Study objectives, outcomes, outcome measures, analysis and results.

Additional data fields may be added to the template allowing for flexibility as required.

\section{Assessment of risk of bias}

Two reviewers (MJ and $\mathrm{KM}$ ) will independently assess the likelihood that components of the design, method or conduct of included studies could lead to misleading or ambiguous results. This will be evaluated using the Effective Public Health Practice Project quality assessment tool for quantitative studies. ${ }^{45}$ This tool was designed to enhance the systematic review process, particularly in the public health arena. It has been assessed to have both content and construct reliability and intra-rater reliability. ${ }^{45}$ The following components will be rated for each study: selection bias, study design, confounders, blinding, data collection methods, withdrawals and dropouts, intervention integrity and data analysis, feasibility and acceptability measures, and process outcomes such as fidelity and reach. Differences in risk of bias ratings in studies will be resolved by discussion between the reviewers. Where agreement cannot be reached, a third reviewer $(\mathrm{ABa})$ will adjudicate.

\section{Data synthesis and analysis}

Due to the overall small size and specificity of the target population, and the likely heterogeneity of treatment interventions and outcome measures, it is anticipated that pooling of study results will be inappropriate, and a narrative synthesis will be undertaken.
Narrative synthesis

A narrative synthesis will be completed following guidelines set out in the 'Cochrane Consumers and Communication Review Group: data synthesis and analysis'. ${ }^{46}$ Accordingly, the following processes will be undertaken:

I. Development of a preliminary synthesis of included studies. A summary of studies and their outcomes will be grouped according to interventional type and tabulated for presentation in the final review. Descriptive statistics will be performed as and where appropriate.

II. A systematic exploration of relationships, both in the data and between studies, to establish similarities and differences in effect sizes between substance types and treatment settings.

III. Assessment of the robustness of the synthesis whereby the quality of the included studies, and of the synthesis methodologies, will be critically appraised.

\section{Patient and public involvement}

Patient or public involvement was not considered appropriate for the development of this protocol as it involves no patient recruitment or use of individual participant data.

\section{Ethics and dissemination}

The review will be a synthesis of research outcomes, with no primary data collection undertaken. As such, ethical approval is not required. Findings will be peer-reviewed and published with all relevant additional materials including search strategies, excluded studies and data extraction. Review outcomes will also be disseminated as conference presentations, in the media and via appropriate social media platforms.

\section{DISCUSSION}

Tobacco use remains a significant problem in some disadvantaged groups of people, including those who are pregnant and dependent on alcohol and/or other psychoactive substances. Within this population, tobacco smoking prevalence remains unacceptably high and targeted treatments are scarce. While earlier reviews have revealed few published studies, the shift in focus towards tobacco cessation treatments for high-priority, disadvantaged groups is increasing the demand for innovative new interventions. This has created a need for the most up-todate evidence related to effective cessation treatments for such populations.

This review will be, to our knowledge, the first comprehensive evaluation of smoking cessation studies targeting maternal populations who experience problems with psychoactive substances. With no restrictions placed on substance type, and no studies excluded due to study design, language or time since publication, this review will identify the extent of current research in this area. The information gained will assist the identification of gaps in our current knowledge and evidence, and highlight 
where and how future treatment resources should be directed.

Twitter Melissa A Jackson @MelJackson_1 and Gillian S Gould @GillianSGould

Acknowledgements We would like to thank Debbie Booth, Senior Librarian at the University of Newcastle for her guidance with the development of the search strategy used in this review.

Contributors $M J$ is the lead and the guarantor of this review. $M J$ and $A B a$ conceptualised the review and drafted the manuscript. $\mathrm{KM}, \mathrm{GG}, \mathrm{ABa}, \mathrm{ABr}$ and $\mathrm{AD}$ provided critical input and were involved in revising the protocol. MJ developed the search strategy included in the protocol. All authors approved the final version of the manuscript and accepted accountability for all aspects of the work.

Funding The authors have not declared a specific grant for this research from any funding agency in the public, commercial or not-for-profit sectors.

Competing interests None declared.

Patient consent for publication Not required.

Provenance and peer review Not commissioned; externally peer reviewed.

Open access This is an open access article distributed in accordance with the Creative Commons Attribution Non Commercial (CC BY-NC 4.0) license, which permits others to distribute, remix, adapt, build upon this work non-commercially, and license their derivative works on different terms, provided the original work is properly cited, appropriate credit is given, any changes made indicated, and the use is non-commercial. See: http://creativecommons.org/licenses/by-nc/4.0/.

\section{ORCID iDs}

Melissa A Jackson http://orcid.org/0000-0002-1491-6218

Amanda L Baker https://orcid.org/0000-0002-3328-7146

Kristen L McCarter http://orcid.org/0000-0002-2638-6381

Amanda L Brown https://orcid.org/0000-0002-0405-0713

Gillian S Gould http://orcid.org/0000-0001-8489-2576

Adrian J Dunlop https://orcid.org/0000-0003-2394-5966

\section{REFERENCES}

1 Kathleen Adams E, Miller VP, Ernst C, et al. Neonatal health care costs related to smoking during pregnancy. Health Econ 2002;11:193-206.

2 US Department of Health and Human Services. Women and smoking: a report of the surgeon General. In: Centre for Disease Control and Prevention, ed, 2001.

3. Castles A, Adams EK, Melvin CL, et al. Effects of smoking during pregnancy. five meta-analyses. Am J Prev Med 1999;16:208-15.

4 Salihu HM, Wilson RE. Epidemiology of prenatal smoking and perinatal outcomes. Early Hum Dev 2007;83:713-20.

5 Al Mamun A, Lawlor DA, Alati R, et al. Does maternal smoking during pregnancy have a direct effect on future offspring obesity? Evidence from a prospective birth cohort study. Am J Epidemiol 2006;164:317-25.

6 Agrawal A, Scherrer JF, Grant JD, et al. The effects of materna smoking during pregnancy on offspring outcomes. Prev Med 2010;50:13-18.

7 Zhou S, Rosenthal DG, Sherman S, et al. Physical, behavioral, and cognitive effects of prenatal tobacco and postnatal secondhand smoke exposure. Curr Probl Pediatr Adolesc Health Care 2014;44:219-41.

8 Magee SR, Bublitz MH, Orazine C, et al. The relationship between Maternal-Fetal attachment and cigarette smoking over pregnancy. Matern Child Health J 2014;18:1017-22.

9 Bernstein IM, Mongeon JA, Badger GJ, et al. Maternal smoking and its association with birth weight. Obstet Gynecol 2005;106:986-91.

10 Lange S, Probst C, Rehm J, et al. National, regional, and global prevalence of smoking during pregnancy in the general population: a systematic review and meta-analysis. Lancet Glob Health 2018;6:e769-76.

11 Chisolm MS, Fitzsimons H, Leoutsakos J-MS, et al. A comparison of cigarette smoking profiles in opioid-dependent pregnant patients receiving methadone or buprenorphine. Nicotine Tob Res 2013;15:1297-304.

12 Jones HE, Heil SH, Tuten M, et al. Cigarette smoking in opioiddependent pregnant women: neonatal and maternal outcomes. Drug Alcohol Depend 2013;131:271-7.
13 Akerman SC, Brunette MF, Green Al, et al. Treating tobacco use disorder in pregnant women in medication-assisted treatment for an opioid use disorder: a systematic review. J Subst Abuse Treat 2015;52:40-7.

14 Fallin A, Miller A, Ashford K. Smoking among pregnant women in outpatient treatment for opioid dependence: a qualitative inquiry. Nicotine Tob Res 2016;18:1727-32.

15 McBride CM, Emmons KM, Lipkus IM. Understanding the potential of teachable moments: the case of smoking cessation. Health Educ Res 2003;18:156-70.

16 Chamberlain C, O'Mara-Eves A, Porter J, et al. Psychosocial interventions for supporting women to stop smoking in pregnancy. Cochrane Database Syst Rev 2017;11.

17. Stead LF, Lancaster T. Combined pharmacotherapy and behavioural interventions for smoking cessation. Cochrane Database Syst Rev 2012.

18 Fiore M, Jaén C, Baker T, et al. Treating tobacco use and dependence: 2008 update [Internet]. Rockville, MD: US Department of Health and Human Services, 2008. Available: https://www.ncbi.nlm.nih.gov/books/NBK63952/ [Accessed 12 Jan 2018].

19 Coleman T, Chamberlain C, Davey MA, et al. Pharmacological interventions for promoting smoking cessation during pregnancy. Cochrane Database Syst Rev 2015;12:.CD010078.

20 Dempsey D, Jacob P, Benowitz NL. Accelerated metabolism of nicotine and cotinine in pregnant smokers. J Pharmacol Exp Ther 2002;301:594-8.

21 Acquavita SP, Talks A, Fiser K. Facilitators and barriers to cigarette smoking while pregnant for women with substance use disorders. Nicotine Tob Res 2017;19:555-61.

22 Chisolm MS, Brigham EP, Lookatch SJ, et al. Cigarette smoking knowledge, attitudes, and practices of patients and staff at a perinatal substance abuse treatment center. J Subst Abuse Treat 2010;39:298-305.

23 Elkader AK, Brands B, Selby P, et al. Methadone-nicotine interactions in methadone maintenance treatment patients. J Clin Psychopharmacol 2009;29:231-8.

24 Gamble J, Grant J, Tsourtos G. Missed opportunities: a qualitative exploration of the experiences of smoking cessation interventions among socially disadvantaged pregnant women. Women Birth 2015;28:8-15.

25 Humfleet GL, Haas AL. Is marijuana use becoming a 'gateway' to nicotine dependence? Addiction 2004;99:5-6.

26 Perez E, Quijano-Cardé N, De Biasi M. Nicotinic mechanisms modulate ethanol withdrawal and modify time course and symptoms severity of simultaneous withdrawal from alcohol and nicotine. Neuropsychopharmacology 2015;40:2327-36.

27 Prochaska JJ. Failure to treat tobacco use in mental health and addiction treatment settings: a form of harm reduction? Drug Alcohol Depend 2010;110:177-82

28 Sharma R, Lodhi S, Sahota P, et al. Nicotine administration in the wake-promoting basal forebrain attenuates sleep-promoting effects of alcohol. J Neurochem 2015;135:323-31.

29 Twyman L, Bonevski B, Paul C, et al. Perceived barriers to smoking cessation in selected vulnerable groups: a systematic review of the qualitative and quantitative literature. BMJ Open 2014;4:e006414.

30 Williams JM, Steinberg ML, Griffiths KG, et al. Smokers with behavioral health comorbidity should be designated a tobacco use disparity group. Am J Public Health 2013;103:1549-55.

31 Williams JM, Ziedonis D. Addressing tobacco among individuals with a mental illness or an addiction. Addict Behav 2004;29:1067-83.

32 Jones HE, Heil SH, O'Grady KE, et al. Smoking in pregnant women screened for an opioid agonist medication study compared to related pregnant and non-pregnant patient samples. Am J Drug Alcohol Abuse 2009;35:375-80.

33 Greaves L, Poole N, Chizimuzo T, et al. Expecting to quit: a bestpractices review of smoking cessation interventions for pregnant and postpartum girls and women [Internet]. 2nd. Vancouver: British Columbia Centre of Excellence for Women's Health, 2011. Available: http://works.bepress.com/chizimuzo_okoli/61/ [Accessed 20 Jan 2019]

34 Tuten M, Fitzsimons $\mathrm{H}$, Chisolm MS, et al. Contingent incentives reduce cigarette smoking among pregnant, methadone-maintained women: results of an initial feasibility and efficacy randomized clinical trial. Addiction 2012;107:1868-77.

35 Holbrook AM, Kaltenbach KA. Effectiveness of a smoking cessation intervention for methadone-maintained women: a comparison of pregnant and parenting women. Int J Pediatr 2011;2011:1-6.

36 Haug NA, Svikis DS, DiClemente C. Motivational enhancement therapy for nicotine dependence in methadone-maintained pregnant women. Psychol Addict Behav 2004;18:289-92. 
37 Ker M, Leischow S, Markowitz IB, et al. Involuntary smoking cessation: a treatment option in chemical dependency programs for women and children. J Psychoactive Drugs 1996;28:47-60.

38 Australian Bureau of Statistics. National health survey: first results, 2014-15, 2015. Available: http://www.abs.gov.au/ausstats/abs@.nsf/ Lookup/by\%20Subject/4364.0.55.001 2014-15 Main\%20Features Smoking 24 [Accessed 23 Jan 2019].

39 Cancer Council Victoria. Smoking \& disadvantage evidence brief [Internet]. Canberra, ACT: Australian National Preventive Health Agency, 2013. Available: https://www.health.gov.au/internet/ publications/publishing.nsf/Content/smoking-disadvantageevidence-brief [Accessed 30 Jan 2019].

40 Moher D, Shamseer L, Clarke M, et al. Preferred reporting items for systematic review and meta-analysis protocols (PRISMA-P) 2015 statement. Syst Rev 2015;4:1.

41 Moher D, Liberati A, Tetzlaff J, et al. Preferred reporting items for systematic reviews and meta-analyses: the PRISMA statement. BMJ 2009;339.
42 Cheung KL, de Ruijter D, Hiligsmann M, et al. Exploring consensus on how to measure smoking cessation. A Delphi study. BMC Public Health 2017;17:890-90.

43 Covidence systematic review software, [program]. Melbourne, Australia: Veritas Health Innovation, 2017. Available: www.covidence. org

44 Higgins JPT, Green S. Cochrane handbook for systematic reviews of interventions [eBook]. Version 5.1. The Cochrane Collaboration, 2011. Available: http://handbook.cochrane.org [Accessed 22 Jan 2019].

45 Thomas BH, Ciliska D, Dobbins $\mathrm{M}$, et al. A process for systematically reviewing the literature: providing the research evidence for public health nursing interventions. Worldviews Evid Based Nurs 2004;1:176-84.

46 Ryan R, Cochrane Consumers and Communication Review Group. Cochrane Consumers and Communication Review Group: data synthesis and analysis [Internet], 2013. Available: http://cccrg. cochrane.org/author-resources [Accessed 22 Jan 2019]. 